\title{
¿Una práctica pedagógica para la sociedad red? Un análisis de la acción docente en el ámbito educativo no universitario.
}

\author{
Josep Maria Mominó \\ jmominoluoc.edu \\ Julio Meneses [1] \\ jmenesesn@uoc.edu \\ Internet Interdisciplinary Institute \\ Universitat Oberta de Catalunya
}

\section{Introducción.}

La importancia del informacionalismo como paradigma tecnológico que actualmente está sustituyendo el industrialismo como matriz dominante de las sociedades del siglo XXI (Castells, 2001a), en el ámbito de la educación escolar, plantea la transición hacia concepciones de la práctica pedagógica capaces de situarse en el nuevo escenario. El reto consiste a evolucionar hacia las opciones con mayor capacidad de incorporar, en el terreno educativo, la potencialidad del nuevo paradigma tecnológico que proporciona la base para la estructuración actual de la sociedad en aquello que se ha denominado "Sociedad Red" (Castells, 2000).

El desarrollo acelerado de las TIC, así como el despliegue vertiginoso de internet en la última década, ha hecho posible la aparición de propuestas educativas que han encontrado en la red oportunidades nuevas y cada vez más diversas para el desarrollo de la práctica educativa. La cuestión es, sin embargo, que algunos analistas (Carnoy, 2004; Smeets, 2004; Cuban, 2003; Cuban, Kirkpatrick y Peck, 2001; para un análisis crítico de la historia de la innovación en el aula con la introducción de tecnología, durante el siglo XX, ver Cuban, 1986, y Cohen, 1987) han empezado a señalar que la innovación que se ha experimentado en el ámbito de la educación escolar con la incorporación de las tecnologías de la información y la comunicación, acaba siendo mucho menos importante que la que podríamos esperar por efecto de la extraordinaria capacidad que ofrecen las TIC para el procesamiento de la información y el trabajo en red. Y es que, el potencial que pueda tener la incorporación de internet en el ámbito educativo para facilitar la transición a que nos referíamos antes (ver, entre otras fuentes, Smeets y Mooij, 2001; Jonassen, 1999; Susman, 1998; Papert, 1993; Cetron y Gayle, 1991), probablemente depende menos de las propias características de las tecnologías como herramientas facilitadoras de la comunicación, la colaboración y la recombinación de la información, que de las características de práctica pedagógica y de las condiciones en que se incorpora su uso (Niederhauser y Stoddart, 2001; Pisapia, 1994; Hannafin y Savenye, 1993).

De acuerdo con este planteamiento, la clave para la interpretación de la potencia de la acción educativa que se desarrolla en las aulas, para dar respuesta a los requerimientos que nos plantea la sociedad actual, a pesar del deslumbramiento que nos produce la tecnología, hay que buscarla, en primer término, en la manera cómo se establece la dinámica entre los componentes fundamentales de la práctica pedagógica (Demetriadis et al., 2003, para un análisis de introducción al contexto griego) y, en segundo término, en la manera diferenciada como se incorpora la red en cada caso (Harrison et al., 2002, para un análisis de introducción en el contexto inglés). La cuestión es que internet, en tanto que instrumento, se puede poner al servicio tanto de los modelos pedagógicos más transmisivos, centrados en la actividad del profesor, como de aquéllos más flexibles que procuran encontrar respuestas ajustadas a la diversidad en que el que aprende encuentra mayores oportunidades de participación activa y de desarrollar la propia autonomía en el la educación propia. 
Entendemos, en este sentido, que los supuestos del profesorado sobre los procesos de enseñanza y aprendizaje y la manera cómo, en último término, configuran los entornos en que desarrollan cotidianamente estos procesos, formas específicas de incorporación de la red. Mejor dicho, favorece determinadas formas de uso a la vez que limita otras, aumentando o disminuyendo a la vez el potencial de la propia práctica para dar respuestas a los requerimientos de una estructura social fundamentada en el informacionalismo y construida, cada vez más, sobre redes de información. Es sobre esta base que justificamos el interés de profundizar en la comprensión de algunos de los rasgos fundamentales de la propia práctica pedagógica con el fin de avanzar en el análisis y la interpretación de los usos específicos de internet en este contexto a que ya nos hemos referido en otro lugar (Mominó, Sigalés, Fornieles, Guasch y Espasa, 2004).

En cambio, no pretendemos detenernos aquí en un análisis complejo de la práctica pedagógica. Se trata, más bien, de ofrecer una representación, de carácter general, de la singularidad de la actividad docente desde la perspectiva de los que la protagonizan: las profesoras y profesores del sistema educativo no universitario de Cataluña. Nos referiremos, pues, al estilo docente en un sentido amplio, haciendo énfasis en tres vertientes que continuamos considerando críticos para una práctica pedagógica que quiera adaptarse a las demandas que nos plantea la sociedad actual: el papel del profesor y el que se concede al alumno en el proceso didáctico; las oportunidades de colaboración que se proporcionan a los que participan en esta dinámica y finalmente, la apertura de la propia actividad educativa a la participación de la comunidad educativa entendida en su sentido más amplio.

\section{Algunas demandas de la Sociedad Red a la educación.}

La rápida evolución hacia la sociedad de la información está produciendo cambios fundamentales en la propia estructura social, tal como la conocemos. La relación que podemos tener con la información y las posibilidades de comunicación de que disponemos están introduciendo modificaciones sustanciales en todos los ámbitos de nuestra sociedad y también en las formas de ofrecer y organizar la educación y, en definitiva, en las formas convencionales de enseñar y aprender, ajustadas muy a menudo a sistemas educativos que, todavía hoy, preparan a las personas para ocupar un lugar en una fábrica u oficina de una sociedad que ya no existe (Hargreaves, 2003; Morin, 2001; Martínez et. al, 2001; Marchesi, 2000; Pérez, 2000).

Las nuevas posibilidades de relación con el conocimiento que se configuran en torno a internet y la Sociedad Red (Castells, 2001c; Lèvy, 1998) ponen de manifiesto la necesidad de ir más allá de la transmisión directa de la información a los alumnos, entendiendo que, más que nunca, el reto se encuentra en la capacidad de aprender y de elaborar el propio conocimiento. Nos encontramos, desde este punto de vista, delante de un "segundo elemento de división social mucho más importante que la conectividad técnica, y es la capacidad educativa y cultural de utilizar internet. Cuándo toda la información está en la red, cuando el conocimiento está en la red, el conocimiento codificado, pero no el conocimiento que se necesita para aquello que se quiere hacer, de lo que se trata es de saber donde está la información, cómo buscarla, cómo procesarla, cómo transformarla en conocimiento específico para aquello que se quiere hacer" (Castells, 2001b).

En una economía progresivamente desarrollada entorno al aprendizaje, es necesario desarrollar la capacidad de procesamiento de la ingente información al alcance para promover su integración en el cuerpo de conocimientos de cada uno (OCDE, 2000). Dada la velocidad de aparición y renovación de los conocimientos existentes en nuestras sociedades, instituciones educativas que limitaran sus funciones a tareas meramente instructivas y transmisivas darían a los alumnos conocimientos, en muchos casos, inminentemente obsoletos. Hoy menos que nunca el aprendizaje no acaba en la escuela, pero es indudable que precisamente los centros educativos, atentos a esta circunstancia, están llamados a desarrollar un papel muy relevante en la capacitación de las personas para la autonomía en su aprendizaje y la orientación a la creación de conocimiento nuevo (Delors, 1996).

La incorporación de las personas a este nuevo marco les plantea la necesidad de desarrollar la autonomía, la capacidad de iniciativa, la proactividad y orientación a la resolución de problemas 
(Hargreaves, 2003). Un aspecto fundamental para el modelo productivo y de relaciones laborales que se está imponiendo en las sociedades de la información consiste, más que nunca, en la capacidad de desarrollar criterios que permitan discernir la relevancia y validez del conocimiento en diferentes situaciones, cuando la aceptación incondicional de la información transmitida desde una fuente única ha perdido su sentido. Asimismo, la transformación de la naturaleza del trabajo se identifica, cada vez más, con la producción de conocimiento a partir de las sinergias establecidas entre diferentes grupos que interaccionan con objetivos compartidos. En este sentido, la escuela tendría que capacitar a sus alumnos para el trabajo habitual en red, es decir, en equipo y en colaboración entre equipos diferentes, de acuerdo con las demandas del mundo laboral del siglo XXI (Carnoy, 2002; Coller, 1997).

En este marco, sobre la base de una concepción del aprendizaje que concede máxima importancia a la actividad del alumno, hay que subrayar que la influencia educativa que ejerce el profesor como mediador entre la actividad constructiva del alumno y los significados que vehiculan los contenidos escolares continúa siendo fundamental (Coll y Onrubia, 1996). Hoy, más que nunca, la acción docente constituye un proceso decisivo de ayuda, capaz de adaptarse a la actividad que necesariamente tiene que protagonizar el alumno. Desde este punto de vista, se pone de manifiesto el interés de acercarnos a la configuración de la acción docente en este proceso, poniendo atención a algunos de los rasgos distintivos de que ya hemos justificado la relevancia: la posición del profesor en el proceso de enseñanza y aprendizaje y la que, en consecuencia, toma al alumno; la capacidad docente de personalización y de adaptación a la diversidad en este proceso; el papel que se otorga a las posibilidades de colaboración con otras personas y, singularmente, con los compañeros; el uso que se hace del material didáctico; y, en último término, la naturaleza atribuida a los procesos de evaluación.

\section{Metodología.}

Para responder a las cuestiones planteadas en esta investigación, nuestro estudio explora la singularidad de la práctica educativa a partir de los datos obtenidos en el "Projecte Internet Catalunya: L'escola a la Societat Xarxa" (Mominó et al, 2004) desarrollado por el Internet Interdisciplinary Instute (IN3) de la Universitat Oberta de Catalunya (UOC), con la financiación de la Generalitat de Catalunya y la colaboración del Departament d'Educació y de la Fundació Jaume Bofill.

Este proyecto de investigación, dirigido por los profesores Josep Maria Mominó y Carles Sigalés, pretende identificar y analizar la incorporación de internet a los centros educativos de Cataluña (educación primaria y secundaria), observando en qué transformaciones está presente y en qué medida contribuye a la aparición de una nueva cultura educativa adaptada a las necesidades que se van configurando en la sociedad informacional. Sin embargo, el interés por un análisis complejo que considere el funcionamiento de los centros educativos, nos ha permitido acercarnos a través de este proyecto de investigación a muchos otros aspectos de la actividad cotidiana del sistema educativo no universitario de forma complementaria. Uno de ellos, la acción docente, es el que nos ocupa en esta investigación, y del que ahora trataremos de hacer una pequeña aproximación presentando los resultados principales.

\subsection{Muestra.}

Por tanto, el universo de estudio está compuesto por el alumnado, el profesorado y los equipos directivos de los 2.726 centros con sede en Cataluña donde se imparten estudios de educación primaria, educación secundaria obligatoria (ESO), bachillerato y ciclos formativos de grado medio (CFGM) o superior, durante el curso 2002 - 2003 de acuerdo a las estadísticas facilitadas por el Departament d'Educació de la Generalitat de Catalunya. Teniendo en cuenta este universo, se fija el tamaño muestral en 350 centros aceptando un margen de error de $\pm 5 \%$ en los resultados globales de los centros, un nivel de confianza del 95,5\% con el supuesto de máxima indeterminación $(\mathrm{p}=\mathrm{q}=50 \mathrm{y}$ $\mathrm{k}=2$ ). Con el objetivo de obtener la máxima representatividad ante la diversidad del universo, el diseño muestral fue aleatorio, estratificado y multietápico, teniendo en cuenta las siguientes variables: niveles o ciclos formativos, distribución territorial, ámbito rural / urbano y titularidad pública / privada. 


\subsection{Instrumentos.}

El trabajo de campo fue realizado entre los meses de diciembre de 2002 y abril de 2003 por un equipo de encuestadores formados específicamente en la administración de estos cuestionarios mediante entrevista personal a un total de 6.612 alumnos (2.918 de primaria, 1.883 de secundaria obligatoria, 1.269 de bachiller y 542 de CFGM o Superior), 2.163 profesores (785 de primaria, 673 de secundaria obligatoria, 533 de bachillerato y 127 de CFGM o Superior), 1.050 responsables de los equipos directivos (525 de primaria, 246 de secundaria obligatoria, 177 de bachillerato y 102 de CFGM o Superior).

De esta forma, los resultados que aquí presentamos se basan en la información recogida a través de una encuesta compuesta por cinco cuestionarios, administrada a una muestra representativa de todos los centros docentes no universitarios de Cataluña (España) a través de una entrevista personal con cada uno de los actores implicados en el funcionamiento de las escuelas (Mominó et al., 2004, para una descripción detallada de la metodología del estudio completo así como del contenido de los cuestionarios). De todos ellos, en la presente investigación nos centramos especialmente en la información proporcionada sobre la práctica educativa por los 350 responsables pedagógicos de etapa y, especialmente, la caracterización que los 2.163 profesores realizan sobre su actividad.

\subsection{Análisis.}

En esta investigación proporcionamos, en primer lugar, una descriptiva de las variables referidas a la caracterización de la práctica pedagógica por parte del profesorado y los responsables pedagógicos de los centros. Para ello, se han categorizado de acuerdo al procedimiento habitual las dimensiones de análisis (elaboración del conocimiento por parte del alumno; participación de los alumnos en el proceso de aprendizaje; participación de padres y otros profesionales en la actividad docente; organización flexible y personalizada de la docencia; trabajo en equipo en la actividad docente; evaluación continua del proceso de enseñanza y aprendizaje; y uso de materiales diversos) originalmente presentadas como ítems de una escala tipo likert de 1 a 10 donde la máxima puntuación supone el mayor acuerdo.

En segundo lugar, para el análisis de diferencias, nos basamos en la comparación de medias sobre cada una de las dimensiones de interés. En este sentido, las pruebas de comparación de medias al uso requieren la utilización de ítems medidos en escalas de intervalo. Sin embargo, en una revisión reciente sobre los riesgos derivados de la violación de este supuesto, Jaccard y Wan (1996) concluyen que el incumplimiento de esta asunción en el tipo de medida no supone ningún riesgo en el caso de los ítems medidos a través de escalas ordinales como los tipo likert de que disponemos (de 1 a 10). De esta forma, se realizan pruebas iniciales de comparación múltiple con el estadístico F (Toothaker, 1991; Miller, 1981) para establecer la existencia de diferencias globales significativas entre las medias con respecto a variables de comparación como la titularidad del centro (público o privado), la etapa en la que desarrolla la acción docente el profesorado (primaria, ESO, bachiller o ciclos formativos), tamaño del centro en función del alumnado total que acoge (variable que surge de la división en tres grupos homogéneos, donde los pequeños tienen menos de 350 alumnos, los medianos entre 350 y 600 , y los grandes más de 600), ubicación del centro (Barcelona y área metropolitana, centros urbanos fuera del área metropolitana, semiurbanos fuera del área metropolitana y ámbito rural), formación inicial del profesorado (técnicos superiores y diplomados, licenciados y tercer ciclo), y edad (variable que surge de la división del profesorado en dos grupos estableciendo el corte en los 35 años de acuerdo con la formulación sobre la estratificación del acceso a las nuevas prácticas de la Sociedad Red en Cataluña de Castells et al., 2003).

Teniendo en cuenta que las condiciones de aplicación de esta prueba de comparación de medias suponen la homogeneidad de las varianzas (Glass, Peckham y Sanders, 1972, para una revisión de las amenazas derivadas de su incumplimiento), de acuerdo al procedimiento habitual utilizamos la prueba de Levene para determinar su incumplimiento. En este caso, en lugar de la prueba de F utilizamos la corrección de Welch, prueba de comparación de medias robusta a la violación de este supuesto 
recomendada en la literatura (Tomarken y Serlin, 1986, para una revisión). A continuación, para las comparaciones múltiples en las que se ven envueltas variables de comparación de más de dos categorías, se establecen las comparaciones correspondientes dos a dos, utilizando en este caso las pruebas post-hoc HSD de Tukey, cuando se cumple la homogeneidad de varianzas, y la corrección de Games-Howell, en caso de su incumplimiento (Toothaker, 1991, para una discusión acerca de los diferentes procedimientos disponibles).

\section{Resultados.}

De esta forma, comenzaremos por revisar las características principales de la práctica pedagógica a partir de la información proporcionada por el propio profesorado (tabla 1) y por los responsables pedagógicos de etapa (tabla 2), que abordaremos a través del estudio del papel de quien estudio y el de quien aprende, el papel de la comunidad, la diversidad como reto, y el empleo de la evaluación al servicio de la mejora.

Tabla 1. Características principales de la práctica pedagógica informadas por el profesorado

\begin{tabular}{|c|c|c|c|c|c|}
\hline & Mucho & Bastante & $\begin{array}{l}\text { Ni bastante ni } \\
\text { poco }\end{array}$ & Poco & Nada \\
\hline $\begin{array}{l}\text { Promoción de la elaboración del conocimiento } \\
\text { por parte del alumno }\end{array}$ & $3,9 \%$ & $21,7 \%$ & $43,3 \%$ & $28,0 \%$ & $3,0 \%$ \\
\hline $\begin{array}{l}\text { Promoción de la participación de los alumnos } \\
\text { en el proceso de aprendizaje }\end{array}$ & $2,5 \%$ & $11,6 \%$ & $31,4 \%$ & $38,3 \%$ & $16,2 \%$ \\
\hline $\begin{array}{l}\text { Promoción de la participación de padres y otros } \\
\text { profesionales en la actividad docente }\end{array}$ & $1,0 \%$ & $6,8 \%$ & $24,8 \%$ & $31,8 \%$ & $35,6 \%$ \\
\hline $\begin{array}{l}\text { Promoción de una organización flexible y } \\
\text { personalizada de la docencia }\end{array}$ & $10,3 \%$ & $28,1 \%$ & $40,2 \%$ & $17,8 \%$ & $3,6 \%$ \\
\hline $\begin{array}{l}\text { Promoción del trabajo en equipo en la actividad } \\
\text { docente }\end{array}$ & $3,4 \%$ & $17,9 \%$ & $44,6 \%$ & $28,5 \%$ & $5,7 \%$ \\
\hline $\begin{array}{l}\text { Promoción de la evaluación continua del } \\
\text { proceso de enseñanza y aprendizaje }\end{array}$ & $32,0 \%$ & $34,8 \%$ & $22,0 \%$ & $8,9 \%$ & $2,2 \%$ \\
\hline $\begin{array}{l}\text { Promoción del uso de materiales diversos } \\
\text { (libros, revistas, materiales multimedia, páginas } \\
\text { web, etc.) }\end{array}$ & $25,6 \%$ & $38,2 \%$ & $25,6 \%$ & $8,6 \%$ & $2,0 \%$ \\
\hline
\end{tabular}

Fuente: elaboración propia $(N=2163)$

Tabla 2. Características principales de las políticas de centro informadas por los responsables pedagógicos

\begin{tabular}{|c|c|c|c|c|c|}
\hline & $\begin{array}{c}\text { No } \\
\text { contemplado }\end{array}$ & En proyecto & $\begin{array}{c}\text { Se está } \\
\text { implementando }\end{array}$ & Muy avanzado & $\begin{array}{c}\text { Objetivo } \\
\text { conseguido }\end{array}$ \\
\hline $\begin{array}{l}\text { Promoción de una docencia más } \\
\text { entrada en la guía del aprendizaje que } \\
\text { en la transmisión de conocimientos }\end{array}$ & $4,9 \%$ & $15,4 \%$ & $50,1 \%$ & $23,8 \%$ & $5,8 \%$ \\
\hline $\begin{array}{l}\text { Promoción de la autonomía del } \\
\text { estudiante en el proceso de } \\
\text { aprendizaje }\end{array}$ & $7,2 \%$ & $7,8 \%$ & $50,1 \%$ & $26,4 \%$ & $8,4 \%$ \\
\hline $\begin{array}{l}\text { Promoción de la flexibilización del los } \\
\text { criterios de agrupación de los alumnos }\end{array}$ & $6,6 \%$ & $9,8 \%$ & $25,6 \%$ & $36,0 \%$ & $21,9 \%$ \\
\hline $\begin{array}{l}\text { Promoción de la flexibilización del } \\
\text { uso del espacio (instalaciones, aulas, } \\
\text { etc.) y el tiempo }\end{array}$ & $6,3 \%$ & $6,9 \%$ & $31,5 \%$ & $35,8 \%$ & $19,5 \%$ \\
\hline
\end{tabular}

Fuente: elaboración propia $(N=350)$

\subsection{El papel de quien enseña y el de quien aprende.}

Poniendo atención, en primer término, a la posición relativa en que se sitúa el profesorado en relación 
al contenido y al papel que se concede al alumno, solamente una cuarta parte $(25,6 \%)$ considera que su acción docente consiste, preferentemente, en promover la elaboración del conocimiento por parte de cada alumno. De hecho, entre éstos, son bien pocos $(3,9 \%)$ los que se sitúan decididamente en esta posición que atribuye máximo protagonismo al estudiante. En el polo contrario, una tercera parte de los profesores (31\%) todavía caracteriza su estilo docente con las formas de docencia más tradicionales, relacionadas con la instrucción directa y la transmisión de información a los alumnos. Esta percepción es confirmada por los responsables pedagógicos de las diferentes etapas educativas: únicamente una tercera parte $(29,6 \%)$ consideran que, en su etapa, ya están muy presentes las formas de docencia más centradas en guiar el aprendizaje del alumno que en procesos de transmisión o de instrucción directa.

En el escenario del informacionalismo y la Sociedad Red (Castells, 2000) las nuevas oportunidades de acceso y procesamiento de la información ponen de manifiesto más que nunca, la importancia de un alto grado de compromiso personal en el proceso de formación y la necesidad de potenciar actitudes y destrezas que permitan el acceso a la información, su recombinación y, en último término, la elaboración del conocimiento. En este sentido, poniendo énfasis en el papel que toma el profesorado en este escenario, en la relación con el contenido y como guía de la actividad que tiene que desarrollar el alumno, es especialmente relevante el valor que se concede a la autonomía del estudiante y las posibilidades que se le ofrecen de intervenir y regular el propio proceso de aprendizaje. En una sociedad fundamentada en el aumento de la capacidad de las personas de procesar la información, dónde este potencial es flexible y accesible desde contextos diversos, las posibilidades de participación y la capacidad de implicación del alumnado en estos procesos de acceso y generación de conocimiento constituye un objetivo pedagógico de primer orden, que se tiene que traducir en la versatilidad del estudiante en su proceso de aprendizaje y en el desarrollo de habilidades que permitan tomar decisiones para adaptar de manera contingente el propio proceso de formación.

La aproximación que nos proporciona al profesorado a esta cuestión permite constatar que son bien pocos $(14,1 \%)$ los que consideran que los alumnos tienen un nivel alto de posibilidades de participación en las decisiones que afectan a su propio proceso de aprendizaje. Entre éstos, son todavía menos $(2,5 \%)$ los que dicen que, de forma cotidiana, en su actividad docente, ofrecen muchas oportunidades en este sentido. Contrariamente, la mayoría $(54,5 \%)$ admiten que conceden pocas opciones para la participación de los estudiantes. La perspectiva más global de los responsables pedagógicos de las diferentes etapas sobre esta cuestión no es mucho más optimista: son pocos ( 8 4\%) los que entienden que ésta es una cuestión ya alcanzada en la etapa y los que consideran que se encuentran en un momento muy avanzado en su incorporación en la práctica son poco más de una cuarta parte $(26,4 \%)$ de estos responsables de etapa.

Desde esta perspectiva, uno de los aspectos críticos de la agenda educativa en la Sociedad Red (OCDE, 2000), el impulso de la capacitación para el aprendizaje permanente y autónomo, parece que todavía tiene que ganar terreno en nuestro sistema educativo. La adquisición de este tipo de metacompetencias requiere de formas de docencia que enseñen como aprender en áreas de conocimiento específicas $y$, en definitiva, que faciliten el desarrollo de un conjunto de "competencias sociocognitivas" (Monereo, 2005), indispensables para la propia evolución en esta sociedad. Entre estas competencias, la capacidad de autorregulación del propio aprendizaje y de tomar decisiones de manera activa en este proceso constituye una destreza fundamental que, según la percepción del propio profesorado a que nos acabamos de referir, todavía tiene una presencia insuficiente en la actividad cotidiana de las aulas.

\subsection{El papel de la comunidad.}

Por otra parte, en un marco en que los límites de la educación formal e informal, entre la escuela y la comunidad, son cada vez más borrosos (Bentley, 1998), los profesores tienden a trabajar, todavía, de forma individualizada y cerrada en su aula con el grupo de alumnos. Los profesores a menudo trabajan solos y saben encontrar pocas oportunidades para la colaboración y la corresponsabilidad de la comunidad educativa. Más de dos terceras partes $(67,4 \%)$ del profesorado indica que en su 
actividad docente hay bien poca o, incluso, ninguna participación por parte de padres o de otros profesionales externos. Son bien pocos los que consideran bastante $(6,8 \%)$ o muy $(1 \%)$ frecuente esta apertura a la participación en su actividad cotidiana.

En este sentido, se ponen de manifiesto las oportunidades que se abren para la escuela en la medida en que sea capaz de llevar a cabo una transición hacia un modelo de trabajo en red que haga posible una mayor implicación de la comunidad en la actividad de la misma aula que todavía se entiende más como un espacio aislado que no como el nodo de una red que tiene que permitir la colaboración y la corresponsabilidad en la elaboración del conocimiento y en la propia acción educativa, en su sentido más amplio. La transición a que nos estamos refiriendo propone desafíos de tipo individual al profesorado, pero también al conjunto del sistema educativo (OCDE, 2000): al profesor le plantea la oportunidad de dejar de trabajar solo para evolucionar hacia una práctica más abierta e interactiva, capaz de incorporar la cooperación con otros profesores del propio centro o de otros a la actividad docente cotidiana, así como de abrirla a la participación de la comunidad educativa. Los desafíos para el sistema educativo también son importantes. Se refieren, de manera prioritaria, a su capacidad de incorporar a la acción educativa escenarios educativos diversos, de favorecer y facilitar la cooperación en red del profesorado para crear, compartir y hacer difusión del conocimiento sobre su actividad profesional.

\subsection{La diversidad como reto.}

El papel central que debe tener el que aprende en una concepción de la práctica pedagógica capaz de responder a los retos que nos plantea a la sociedad actual, pone de manifiesto que la calidad de esta práctica tenga que vincularse a la capacidad docente de ajustarse a la singularidad del proceso de aprendizaje que sigue a cada alumno. La mejora indudable que ha supuesto para nuestra sociedad la ampliación de la escolaridad obligatoria sobre la base del principio de comprensividad ha puesto sobre la mesa el reto de la atención a la diversidad. Para responder a este reto, ante la necesidad de promover el desarrollo de competencias y actitudes necesarias en el marco de autoresponsabilidad a que nos estamos refiriendo, la eficacia del proceso de enseñanza y aprendizaje continúa dependiendo, en gran medida, del despliegue, en la docencia, de formas flexibles de organización de la actividad del aula y, en último término, de estrategias personalizadas de apoyo y guía.

A pesar del valor que le concedamos, sin embargo, la inclinación de la acción docente hacia este tipo de planteamientos todavía no parece mayoritaria $(38,4 \%)$ : solamente uno de cada diez profesores $(10,3 \%)$ asegura que la organización flexible y personalizada es un aspecto muy característico de su docencia. Los que incorporan menos, pero todavía a menudo, esta diversificación de actividades son poco más de la cuarta parte $(28,1 \%)$ del profesorado. La cuestión es, sin embargo, que un conjunto importante $(40,2 \%)$ de profesores, cuando tienen que calibrar la medida en que la personalización es definitoria de su actuación en el aula, se sitúan en una prudente posición intermedia y, en último término, dos de cada diez profesores $(21,4 \%)$ todavía dicen que mantienen formas de actividad que buscan la homogeneidad y procuran mantener ritmos iguales para todos.

Hay que decir, sin embargo, que la importancia que conceden los centros a la personalización de la acción educativa se pone de manifiesto cuando nos fijamos en la manera como se potencian los aspectos de organización que tendrían que facilitar esta capacidad de adaptación a la diversidad de ritmos de aprendizaje que se producen en cualquier aula: más de la mitad de los responsables pedagógicos de las diferentes etapas $(57,9 \%)$ consideran que la flexibilidad en los criterios de agrupamiento de los alumnos es un aspecto en que su etapa ha avanzado significativamente. Esta perspectiva se mantiene $(55,3 \%)$ cuando se refieren a la medida en que consideran que se ha progresado en la flexibilización del uso del espacio y del tiempo para estos mismos propósitos.

No obstante, la respuesta a la heterogeneidad depende, en gran medida, de la capacidad de impulsar formas flexibles de organización de la actividad del aula que sepan incorporar estratégicamente el potencial de la propia diversidad. Para este propósito, son fundamentales las oportunidades que ofrece la interacción entre los propios alumnos y, en concreto, el trabajo cooperativo como "modalidad de 
organización social del aula en que los alumnos tienen que colaborar para conseguir realizar la tarea de aprendizaje" (Marchesi y Martín, 1998, pp. 346-350). La representación que nos proporciona al profesorado de la forma de actividad que impulsa habitualmente en su aula se corresponde poco con una estructura general de cooperación: más de una tercera parte del profesorado $(34,2 \%)$ nos dice que no promueve el trabajo en equipo de los alumnos o que lo hace bien poco. De hecho, los que consideran habitual esta forma de trabajo en la actividad de su aula no llegan a la cuarta parte de los profesores $(21,3 \%)$.

Entendiendo que el trabajo individual es insustituible para asegurar la elaboración personal del conocimiento, su preeminencia en esta representación de la práctica pone de manifiesto las dificultades de los centros para incorporar el potencial de la cooperación en la actividad cotidiana de las aulas. No es éste el lugar para profundizar en la idea de aprendizaje cooperativo, pero hay que subrayar que un planteamiento fundamentalmente individualista como el que parece que se produce, supone dejar de lado las oportunidades que ofrece la organización social de la actividad para la motivación, el tipo de implicación de los alumnos en el proceso de aprendizaje y para su desarrollo social. Hay que decir, sin embargo, que aunque la responsabilidad de impulsar este tipo de trabajo recae en último término en manos del profesorado, requiere de estructuras organizativas que hagan posible la gestión del aula de manera que pueda ser abordada con garantías de eficiencia como estrategia fundamental de atención a la diversidad.

\subsection{La evaluación al servicio de la mejora.}

En el compromiso de la educación con los parámetros que la tienen que situar adecuadamente ante las exigencias de la Sociedad Red, la evaluación continúa siendo un instrumento fundamental de gestión de la calidad. La función pedagógica de la evaluación a qua ahora nos referimos, más allá de constatar resultados, tiene que permitir adaptar el proceso de enseñanza y aprendizaje con el propósito que éste se produzca de la manera más eficiente posible. Este planteamiento comporta una mecánica continua, referida principalmente al proceso, más que al producto, que tiene que proporcionar elementos que permitan poner de manifiesto en que medida el aprendizaje se está produciendo de la manera más pertinente posible $\mathrm{y}$, en último término, activar los mecanismos de ajuste necesarios para la personalización imprescindible a que nos referíamos antes.

Del análisis que hemos podido hacer de este aspecto, se desprende que esta función formativa o reguladora de la evaluación se ha incorporado en buena medida en la práctica docente cotidiana: dos terceras partes del profesorado $(66,8 \%)$ aseguran que evalúan de manera continuada la actividad de enseñanza y aprendizaje como instrumento de mejora de este mismo proceso. Son bien pocos $(11,1 \%)$ los que utilizan la evaluación prioritariamente como instrumento para la identificación del resultado final en el rendimiento de los alumnos. Más allá del valor indudable de esta función sumativa, la situación con que nos encontramos, parece proclive a la obtención continua de información para el diagnóstico de las situaciones educativas, necesariamente diversas y personalizadas. En este sentido, tiene una alta potencialidad para la capacidad de tomar decisiones sobre la articulación del proceso pedagógico y, en definitiva, para la mejora de su calidad. La cuestión es, sin embargo, que la capacidad de procesamiento, recombinación y comunicación de la información que nos proporcionan las TIC no parece que se haya incorporado al ámbito educativo, en contraste con otros sectores, ni para el análisis del progreso de los alumnos, ni para la gestión de la calidad de sus resultados (Carnoy, 2004).

Tal como apuntan otros estudios (OCDE, 2001), hay que poner énfasis una vez más, en la necesidad de una actividad pedagógica más interdisciplinaria, ajustada, en este sentido, a la vida real que se pueda desarrollar y acreditar a través de procesos de evaluación formativos y acumulativos. Es en este tipo de procesos en los que las TIC y la propia red proporcionan un instrumento potente para la mejora educativa sistemática. Este potencial, no obstante, puede ser aprovechado de manera limitada cuando se aplica sobre la base de los procedimientos de evaluación más tradicionales. El escenario en que nos encontramos ofrecería oportunidades en este sentido pero, en cambio, parece que hemos avanzado poco en esta dirección, probablemente por la existencia de algunos obstáculos importantes 
como la falta de conocimientos del profesorado para el uso de instrumentos de análisis de datos (Carnoy, 2004).

\section{5. ¿Una práctica heterogénea?}

Sin ningún tipo de duda, la configuración de la práctica pedagógica es compleja y se traduce en experiencias muy diversas, derivadas de la singularidad de cada centro y, probablemente, de las peculiaridades de la acción docente de cada profesor. Sin embargo, en la representación que nos ofrece el profesorado (ver tabla 3), las diferencias que se observan en las grandes tendencias son, generalmente, poco importantes.

\section{Tabla 3. Medias y desviaciones estándar de las características principales de la práctica pedagógica informadas por el profesorado}

\begin{tabular}{|l|c|c|}
\hline & Media & Std. Dev. \\
\hline Promoción de la elaboración del conocimiento por parte del alumno & 4,84 & 2,12 \\
\hline $\begin{array}{l}\text { Promoción de la participación de los alumnos en el proceso de } \\
\text { aprendizaje }\end{array}$ & 3,66 & 2,25 \\
\hline $\begin{array}{l}\text { Promoción de la participación de padres y otros profesionales en la } \\
\text { actividad docente }\end{array}$ & 2,77 & 2,23 \\
\hline Promoción de una organización flexible y personalizada de la docencia & 5,58 & 2,32 \\
\hline Promoción del trabajo en equipo en la actividad docente & 4,59 & 2,14 \\
\hline $\begin{array}{l}\text { Promoción de la evaluación continua del proceso de enseñanza y } \\
\text { aprendizaje }\end{array}$ & 7,01 & 2,36 \\
\hline $\begin{array}{l}\text { Promoción del uso de materiales diversos (libros, revistas, materiales } \\
\text { multimedia, páginas web, etc.) }\end{array}$ & 6,86 & 2,30 \\
\hline
\end{tabular}

Fuente: elaboración propia $(N=2163)$

Procuraremos, sin embargo, identificar los matices y nos referiremos, por una parte, a algunos de los principales aspectos que distinguen globalmente el conjunto de los centros y, por otra, a algunos de los factores que diferencian al profesorado.

\subsubsection{Diferencias en función de la titularidad.}

Poniendo atención, primeramente, en algunos de los rasgos que permiten distinguir el conjunto de los centros educativos, hay que subrayar que la titularidad de los centros no parece ser un factor determinante de las formas de acción docente (ver tabla 4). No hay discrepancias significativas entre los profesores de los centros públicos y privados cuando se refieren a las oportunidades que conceden a los alumnos de implicarse activamente en su proceso de aprendizaje. Tampoco las encontramos cuando nos fijamos en la capacidad docente de personalización y adaptación a las diferencias individuales. Tal como decíamos antes, los estilos docentes más centrados en el profesor, dirigidos a la transmisión directa de contenidos, todavía tienen una presencia bastante destacada. En este aspecto, unos y otros se sitúan en una posición similar.

Tabla 4. Comparación de medias en función de la titularidad del centro

$$
\text { (G1 = titularidad pública; G2 = titularidad privada) }
$$

\begin{tabular}{|l|c|c|c|}
\hline & $\begin{array}{c}\text { Media G1 (std. } \\
\text { dev.) }\end{array}$ & $\begin{array}{c}\text { Media G2 (std. } \\
\text { dev.) }\end{array}$ & Diferencia de medias* \\
\hline $\begin{array}{l}\text { Promoción de la elaboración del conocimiento por parte } \\
\text { del alumno }\end{array}$ & $4,85(2,15)$ & $4,82(2,07)$ & $\mathrm{F}=0,131$ \\
\hline $\begin{array}{l}\text { Promoción de la participación de los alumnos en el } \\
\text { proceso de aprendizaje }\end{array}$ & $3,84(2,28)$ & $3,39(2,19)$ & $\mathrm{F}=20,855^{\mathrm{a}}$ \\
\hline $\begin{array}{l}\text { Promoción de la participación de padres y otros } \\
\text { profesionales en la actividad docente }\end{array}$ & $2,87(2,27)$ & $2,61(2,16)$ & $\mathrm{F}=6,866^{\mathrm{b}}$ \\
\hline Promoción de una oroanización flexihle v nersonalizada & & &
\end{tabular}




\begin{tabular}{|c|c|c|c|}
\hline de la docencia & $5,55(2,37)$ & $5,62(2,24)$ & $\mathrm{W}=0,478$ \\
\hline Promoción del trabajo en equipo en la actividad docente & $4,74(2,16)$ & $4,35(2,09)$ & $\mathrm{F}=16,957^{\mathrm{a}}$ \\
\hline $\begin{array}{l}\text { Promoción de la evaluación continua del proceso de } \\
\text { enseñanza y aprendizaje }\end{array}$ & $6,91(2,36)$ & $7,17(2,34)$ & $\mathrm{F}=6,053^{\mathrm{b}}$ \\
\hline $\begin{array}{l}\text { Promoción del uso de materiales diversos (libros, } \\
\text { revistas, materiales multimedia, páginas web, etc.) }\end{array}$ & $6,90(2,29)$ & $6,79(2,32)$ & $\mathrm{F}=1,165$ \\
\hline \multicolumn{4}{|c|}{$\begin{array}{l}\text { * En caso de incumplimiento del supuesto de homogeneidad de medias necesario para la aplicación de la prueba } \mathrm{F} \text { (prueb } \\
\text { de Levene significativa con una } \mathrm{p} \leq 0.05 \text { ), se aplica la prueba de comparación de medias con la corrección de Welch (W). }\end{array}$} \\
\hline \multicolumn{4}{|l|}{ a Diferencias significativas con una $\mathrm{p} \leq 0.001$} \\
\hline \multicolumn{4}{|l|}{${ }^{b}$ Diferencias significativas con una $\mathrm{p} \leq 0.010$} \\
\hline${ }^{c}$ Diferencias significativas con una $\mathrm{p} \leq 0.050$ & & & \\
\hline
\end{tabular}

Fuente: elaboración propia $(N=2163)$

De hecho, cuando encontramos diferencias, se producen en favor de la escuela pública, salvo en el caso de las formas de evaluación que, desde la óptica del profesorado, se desarrollarían en su vertiente más formativa en la escuela privada. Las diferencias que se identifican en otros aspectos de la docencia podríamos decir que dejan en mejor posición los centros públicos. Éste es el caso de las opciones que se ofrecen a los alumnos para tomar decisiones sobre su propio proceso de aprendizaje, de la presencia del trabajo en equipo, de las formas de docencia más abiertas y con mayor disposición a la participación externa de padres y otros profesionales. En todos estos aspectos, las diferencias, sin ser muy amplias, son significativas e indican, para al caso de la titularidad pública, una posición docente con mayor potencialidad de adaptación a los requerimientos de una sociedad como la nuestra.

\subsubsection{Diferencias en función de la etapa impartida.}

Por otra parte, cuando nos fijamos en la etapa educativa, las diferencias se establecen principalmente entre el profesorado de primaria y el del resto de etapas (ver tablas 5a y 5b). Es en esta primera etapa y, en algunos casos, también en los ciclos formativos, donde encontramos los planteamientos más centrados en el alumno: los profesores de primaria se distancian significativamente del resto de etapas y, principalmente del bachillerato, en la adopción de las posiciones que más pretenden favorecer el protagonismo del estudiante en la elaboración del propio conocimiento.

\section{Tabla 5a. Comparación de medias en función de la etapa impartida}

$$
\text { (G1 = primaria; G2 = ESO; G3 = bachillerato; } \mathbf{G 4} \text { = ciclos formativos })
$$

\begin{tabular}{|c|c|c|c|c|c|}
\hline & $\begin{array}{l}\text { Media G1 } \\
\text { (std. dev.) }\end{array}$ & $\begin{array}{l}\text { Media G2 } \\
\text { (std. dev.) }\end{array}$ & $\begin{array}{l}\text { Media G3 } \\
\text { (std. dev.) }\end{array}$ & $\begin{array}{l}\text { Media G4 } \\
\text { (std. dev.) }\end{array}$ & Diferencia de medias* \\
\hline $\begin{array}{l}\text { Promoción de la elaboración del } \\
\text { conocimiento por parte del alumno }\end{array}$ & $5,18(2,19)$ & $4,74(1,99)$ & $4,54(2,08)$ & $4,61(2,17)$ & $\mathrm{W}=11,057^{\mathrm{a}}$ \\
\hline $\begin{array}{l}\text { Promoción de la participación de los } \\
\text { alumnos en el proceso de aprendizaje }\end{array}$ & $3,87(2,33)$ & $3,40(2,19)$ & $3,65(2,19)$ & $3,81(2,26)$ & $\mathrm{F}=5,528^{\mathrm{a}}$ \\
\hline $\begin{array}{l}\text { Promoción de la participación de padres } \\
\text { y otros profesionales en la actividad } \\
\text { docente }\end{array}$ & $3,38(2,27)$ & $2,35(2,06)$ & $2,22(2,08)$ & $3,29(2,32)$ & $\mathrm{W}=41,971^{\mathrm{a}}$ \\
\hline $\begin{array}{l}\text { Promoción de una organización flexible } \\
\text { y personalizada de la docencia }\end{array}$ & $6,13(2,18)$ & $5,68(2,22)$ & $4,75(2,42)$ & $5,28(2,28)$ & $\mathrm{W}=38,051^{\mathrm{a}}$ \\
\hline $\begin{array}{l}\text { Promoción del trabajo en equipo en la } \\
\text { actividad docente }\end{array}$ & $4,99(2,06)$ & $4,33(2,06)$ & $4,04(2,13)$ & $5,45(2,24)$ & $\mathrm{F}=34,696^{\mathrm{a}}$ \\
\hline $\begin{array}{l}\text { Promoción de la evaluación continua del } \\
\text { proceso de enseñanza y aprendizaje }\end{array}$ & $7,59(2,14)$ & $7,04(2,24)$ & $6,22(2,58)$ & $6,68(2,31)$ & $\mathrm{W}=36,039^{\mathrm{a}}$ \\
\hline Promoción del uso de ma & $7,19(2,14)$ & $6,60(2,35)$ & $6,52(2,41)$ & $7,42(2,18)$ & $\mathrm{W}=15.841^{\mathrm{a}}$ \\
\hline
\end{tabular}


diversos (libros, revistas, materiales multimedia, páginas web, etc.)

* En caso de incumplimiento del supuesto de homogeneidad de medias necesario para la aplicación de la prueba $\mathrm{F}$ (prueba de Levene significativa con una $\mathrm{p} \leq 0.05)$, se aplica la prueba de comparación de medias con la corrección de Welch (W).

Diferencias significativas con una $\mathrm{p} \leq 0.001$

Diferencias significativas con una $\mathrm{p} \leq 0.010$

Diferencias significativas con una $\mathrm{p} \leq 0.050$

Fuente: elaboración propia $(N=2163)$

Siguiendo esta tendencia, también es en el primer tramo de la educación obligatoria donde parece que la docencia se diversifica en mayor grado con el propósito de ajustarse las diferencias y ritmos individuales. También, en este caso, es en la educación postobligatoria donde encontramos planteamientos docentes menos flexibles y una mayor inclinación a la uniformidad. Seguramente, eso explica también que sea en primaria y en los ciclos formativos donde hay una mayor tendencia a incorporar materiales diversos en la actividad cotidiana del aula con el fin de poder llevar a cabo estas formas de acción docente. Por comparación, en la educación secundaria, tanto en su tramo obligatorio como post-obligatorio, los profesores se acercan con más frecuencia al uso del libro de texto como único material.

Asimismo, de manera coherente con este planteamiento, los procesos de evaluación continuada con más capacidad de ajustarse al alumno, los que tienen un carácter más formativo, tienen también mayor presencia en la educación primaria que en la secundaria. En el bachillerato, incluso en comparación con la ESO, es donde el profesorado tiende más a utilizar la evaluación únicamente como instrumento final de medida del rendimiento de los alumnos, más que para la mejora de los procesos de aprendizaje y de la propia docencia. Es más, el mayor protagonismo del alumno que estamos asociando a diferentes aspectos de la docencia en la educación primaria se confirma en la comparación con la ESO cuando ponemos la atención en las posibilidades que se les ofrecen de tomar determinaciones que afectan a su proceso de aprendizaje. En este caso, sin embargo, las diferencias con el resto de etapas no son significativas.

Más allá del lugar que encuentra el estudiante en la actividad que se produce en las aulas, si nos fijamos en la manera como se promueve la colaboración y la participación, nuevamente es en el primer tramo de la educación obligatoria y en los ciclos formativos donde el profesorado dice que potencia más el trabajo en equipo y que incorpora en mayor medida la participación de la comunidad educativa. También en estos aspectos hay diferencias significativas en la comparación con las posiciones docentes de la educación secundaria.

Tabla 5b. Pruebas post-hoc para la comparación de medias dos a dos en función de la etapa impartida*

\begin{tabular}{|c|c|c|c|}
\hline & Etapa (I) & Etapa $(\mathbf{J})$ & Dif. de medias (I-J) \\
\hline \multirow{6}{*}{$\begin{array}{l}\text { Promoción de la elaboración del conocimiento por parte } \\
\text { del alumno (Prueba de Games Howell) }\end{array}$} & \multirow{3}{*}{ Primaria } & ESO & $0,440^{\mathrm{a}}$ \\
\hline & & Bachillerato & $0,644^{\mathrm{a}}$ \\
\hline & & Ciclos formativos & $0,567^{\mathrm{b}}$ \\
\hline & \multirow{2}{*}{ ESO } & Bachillerato & 0,204 \\
\hline & & Ciclos formativos & 0,127 \\
\hline & Bachillerato & Ciclos formativos & $-0,078$ \\
\hline \multirow{4}{*}{$\begin{array}{l}\text { Promoción de la participación de los alumnos en el } \\
\text { proceso de aprendizaje (Prueba HSD de Tukey) }\end{array}$} & \multirow{3}{*}{ Primaria } & ESO & $0,473^{\mathrm{a}}$ \\
\hline & & Bachillerato & 0,223 \\
\hline & & Ciclos formativos & 0,059 \\
\hline & ESO & Bachillerato & $-0,250$ \\
\hline
\end{tabular}




\begin{tabular}{|c|c|c|c|}
\hline & & Ciclos formativos & $-0,414$ \\
\hline & Bachillerato & Ciclos formativos & $-0,164$ \\
\hline \multirow{6}{*}{$\begin{array}{l}\text { Promoción de la participación de padres y otros } \\
\text { profesionales en la actividad docente (Prueba de Games } \\
\text { Howell) }\end{array}$} & \multirow{3}{*}{ Primaria } & ESO & $1,024^{\mathrm{a}}$ \\
\hline & & Bachillerato & $1,156^{\mathrm{a}}$ \\
\hline & & Ciclos formativos & 0,092 \\
\hline & \multirow{2}{*}{ ESO } & Bachillerato & 0,131 \\
\hline & & Ciclos formativos & $-0,933^{a}$ \\
\hline & Bachillerato & Ciclos formativos & $-1,064^{a}$ \\
\hline \multirow{6}{*}{$\begin{array}{l}\text { Promoción de una organización flexible y personalizada } \\
\text { de la docencia (Prueba de Games Howell) }\end{array}$} & \multirow{3}{*}{ Primaria } & ESO & $0,450^{\mathrm{a}}$ \\
\hline & & Bachillerato & $1,379^{\mathrm{a}}$ \\
\hline & & Ciclos formativos & $0,851^{\mathrm{a}}$ \\
\hline & \multirow{2}{*}{ ESO } & Bachillerato & $0,928^{\mathrm{a}}$ \\
\hline & & Ciclos formativos & 0,401 \\
\hline & Bachillerato & Ciclos formativos & $-0,527^{\mathrm{c}}$ \\
\hline \multirow{6}{*}{$\begin{array}{l}\text { Promoción del trabajo en equipo en la actividad docente } \\
\text { (Prueba HSD de Tukey) }\end{array}$} & \multirow{3}{*}{ Primaria } & ESO & $0,662^{\mathrm{a}}$ \\
\hline & & Bachillerato & $0,950^{\mathrm{a}}$ \\
\hline & & Ciclos formativos & $-0,461^{\mathrm{c}}$ \\
\hline & \multirow{2}{*}{ ESO } & Bachillerato & 0,288 \\
\hline & & Ciclos formativos & $-1,122^{\mathrm{a}}$ \\
\hline & Bachillerato & Ciclos formativos & $-1,410^{\mathrm{a}}$ \\
\hline \multirow{6}{*}{$\begin{array}{l}\text { Promoción de la evaluación continua del proceso de } \\
\text { enseñanza y aprendizaje (Prueba de Games Howell) }\end{array}$} & \multirow{3}{*}{ Primaria } & ESO & $0,554^{\mathrm{a}}$ \\
\hline & & Bachillerato & $1,373^{\mathrm{a}}$ \\
\hline & & Ciclos formativos & $0,909^{\mathrm{a}}$ \\
\hline & \multirow{2}{*}{ ESO } & Bachillerato & $0,819^{\mathrm{a}}$ \\
\hline & & Ciclos formativos & 0,355 \\
\hline & Bachillerato & Ciclos formativos & $-0,464$ \\
\hline \multirow{6}{*}{$\begin{array}{l}\text { Promoción del uso de materiales diversos (libros, } \\
\text { revistas, materiales multimedia, páginas web, etc.) } \\
\text { (Prueba de Games Howell) }\end{array}$} & \multirow{3}{*}{ Primaria } & ESO & $0,589^{\mathrm{a}}$ \\
\hline & & Bachillerato & $0,669^{\mathrm{a}}$ \\
\hline & & Ciclos formativos & $-0,226$ \\
\hline & \multirow{2}{*}{ ESO } & Bachillerato & $0,080^{\mathrm{a}}$ \\
\hline & & Ciclos formativos & $-0,815$ \\
\hline & Bachillerato & Ciclos formativos & $-0,895^{\mathrm{a}}$ \\
\hline \multicolumn{4}{|c|}{$\begin{array}{l}\text { * En caso de incumplimiento del supuesto de homogeneidad de medias necesario para la aplicación de la prueba HSD de } \\
\text { Tukey (prueba de Levene significativa con una } \mathrm{p} \leq 0.05 \text { ), se aplica la prueba de comparación de medias con la corrección de } \\
\text { Games-Howell }(\mathrm{GH}) \text {. Se han eliminado las comparaciones redundantes. }\end{array}$} \\
\hline \multicolumn{4}{|l|}{ a Diferencias significativas con una $\mathrm{p} \leq 0.001$} \\
\hline \multicolumn{4}{|l|}{$\mathrm{b}$ Diferencias significativas con una $\mathrm{p} \leq 0.010$} \\
\hline Diferencias significativas con una $\mathrm{p} \leq 0.050$ & & & \\
\hline
\end{tabular}

Fuente: elaboración propia $(N=2163)$

\subsubsection{Diferencias en función del tamaño y la ubicación de los centros.}

El tamaño de los centros (ver tablas 6a y 6b) y su ubicación (ver tablas 7a y 7b) también podrían tener alguna incidencia en la configuración de la práctica educativa. Así, con respecto al tamaño, cuando comparamos la posición que toman los profesores de los centros mayores con la de los más pequeños (de menos de trescientos cincuenta alumnos) se adivina que, en éstos últimos, probablemente, encuentran condiciones que les facilitan una práctica docente más personalizada. En este sentido, se incorpora en mayor medida la evaluación, en el su vertiente formativa, como instrumento ajustado a la mejora de cada proceso de enseñanza y aprendizaje, se ofrecen más oportunidades a los alumnos de 
decidir sobre las cuestiones que afectan su aprendizaje, se aprecia una mayor tendencia a promover dinámicas de colaboración y trabajo en equipo y, en último término, la práctica docente parece que se abre con más facilidad a la participación de la comunidad educativa.

Tabla 6a. Comparación de medias en función del tamaño del centro

$$
\text { (G1 = pequeño; G2 = mediano; G3 = grande })
$$

\begin{tabular}{|c|c|c|c|c|}
\hline & $\begin{array}{l}\text { Media G1 } \\
\text { (std. dev.) } \\
\end{array}$ & $\begin{array}{c}\text { Media G2 (std. } \\
\text { dev.) }\end{array}$ & $\begin{array}{c}\text { Media G3 (std. } \\
\text { dev.) }\end{array}$ & $\begin{array}{l}\text { Diferencia de } \\
\text { medias* }\end{array}$ \\
\hline $\begin{array}{l}\text { Promoción de la elaboración del conocimiento por parte } \\
\text { del alumno }\end{array}$ & $4,91(2,09)$ & $4,84(2,15)$ & $4,77(2,11)$ & $\mathrm{F}=0,852$ \\
\hline $\begin{array}{l}\text { Promoción de la participación de los alumnos en el } \\
\text { proceso de aprendizaje }\end{array}$ & $3,88(2,29)$ & $3,67(2,27)$ & $3,43(2,18)$ & $\mathrm{F}=6,840^{\mathrm{a}}$ \\
\hline $\begin{array}{l}\text { Promoción de la participación de padres y otros } \\
\text { profesionales en la actividad docente }\end{array}$ & $3,08(2,33)$ & $2,66(2,18)$ & $2,56(2,14)$ & $\mathrm{W}=10,618^{\mathrm{a}}$ \\
\hline $\begin{array}{l}\text { Promoción de una organización flexible y personalizada de } \\
\text { la docencia }\end{array}$ & $5,87(2,29)$ & $5,52(2,38)$ & $5,33(2,26)$ & $\mathrm{F}=9,982^{\mathrm{a}}$ \\
\hline Promoción del trabajo en equipo en la actividad docente & $4,93(2,10)$ & $4,56(2,15)$ & $4,26(2,13)$ & $\mathrm{F}=17,304^{\mathrm{a}}$ \\
\hline $\begin{array}{l}\text { Promoción de la evaluación continua del proceso de } \\
\text { enseñanza y aprendizaje }\end{array}$ & $7,23(2,16)$ & $6,93(2,42)$ & $6,87(2,48)$ & $\mathrm{W}=5,211^{\mathrm{b}}$ \\
\hline $\begin{array}{l}\text { Promoción del uso de materiales diversos (libros, revistas, } \\
\text { materiales multimedia, páginas web, etc.) }\end{array}$ & $7,06(2,28)$ & $6,74(2,33)$ & $6,78(2,28)$ & $\mathrm{F}=4,352^{\mathrm{b}}$ \\
\hline \multicolumn{5}{|c|}{$\begin{array}{l}\text { * En caso de incumplimiento del supuesto de homogeneidad de medias necesario para la aplicación de la prueba } \mathrm{F} \text { (prueba } \\
\text { de Levene significativa con una } \mathrm{p} \leq 0.05 \text { ), se aplica la prueba de comparación de medias con la corrección de Welch (W). }\end{array}$} \\
\hline \multicolumn{5}{|l|}{ a Diferencias significativas con una $\mathrm{p} \leq 0.001$} \\
\hline \multicolumn{5}{|l|}{${ }^{b}$ Diferencias significativas con una $p \leq 0.010$} \\
\hline${ }^{\mathrm{c}}$ Diferencias significativas con una $\mathrm{p} \leq 0.050$ & & & & \\
\hline
\end{tabular}

Fuente: elaboración propia $(N=2163)$

Tabla 6b. Pruebas post-hoc para la comparación de medias dos a dos en función del tamaño del centro*

\begin{tabular}{|c|c|c|c|}
\hline & Tamaño (I) & Tamaño (J) & Dif. de medias (I-J) \\
\hline \multirow{3}{*}{$\begin{array}{l}\text { Promoción de la elaboración del conocimiento por parte del } \\
\text { alumno (Prueba HSD de Tukey) }\end{array}$} & \multirow{2}{*}{ Pequeño } & Mediano & 0,077 \\
\hline & & Grande & 0,147 \\
\hline & Mediano & Grande & 0,070 \\
\hline \multirow{3}{*}{$\begin{array}{l}\text { Promoción de la participación de los alumnos en el proceso de } \\
\text { aprendizaje (Prueba HSD de Tukey) }\end{array}$} & \multirow{2}{*}{ Pequeño } & Mediano & 0,208 \\
\hline & & Grande & $0,443^{\mathrm{a}}$ \\
\hline & Mediano & Grande & 0,235 \\
\hline \multirow{3}{*}{$\begin{array}{l}\text { Promoción de la participación de padres y otros profesionales } \\
\text { en la actividad docente (Prueba de Games Howell) }\end{array}$} & \multirow{2}{*}{ Pequeño } & Mediano & $0,420^{\mathrm{a}}$ \\
\hline & & Grande & $0,522^{a}$ \\
\hline & Mediano & Grande & 0,101 \\
\hline \multirow{3}{*}{$\begin{array}{l}\text { Promoción de una organización flexible y personalizada de la } \\
\text { docencia (Prueba HSD de Tukey) }\end{array}$} & \multirow{2}{*}{ Pequeño } & Mediano & $0,351^{b}$ \\
\hline & & Grande & $0,542^{a}$ \\
\hline & Mediano & Grande & 0,192 \\
\hline \multirow{3}{*}{$\begin{array}{l}\text { Promoción del trabajo en equipo en la actividad docente } \\
\text { (Prueba HSD de Tukey) }\end{array}$} & \multirow{2}{*}{ Pequeño } & Mediano & $0,360^{\mathrm{b}}$ \\
\hline & & Grande & $0,664^{\mathrm{a}}$ \\
\hline & Mediano & Grande & $0,303^{\mathrm{c}}$ \\
\hline \multirow{3}{*}{$\begin{array}{l}\text { Promoción de la evaluación continua del proceso de enseñanza } \\
\text { y aprendizaje (Prueba de Games Howell) }\end{array}$} & \multirow{2}{*}{ Pequeño } & Mediano & $0,306^{\mathrm{c}}$ \\
\hline & & Grande & $0,360^{b}$ \\
\hline & Mediano & Grande & 0,054 \\
\hline
\end{tabular}


Promoción del uso de materiales diversos (libros, revistas, materiales multimedia, páginas web, etc.) (Prueba HSD de Tukey)

\begin{tabular}{|c|c|c|}
\hline \multirow{2}{*}{ Pequeño } & Mediano & $0,327^{\mathrm{c}}$ \\
\cline { 2 - 3 } & Grande & $0,289^{\mathrm{c}}$ \\
\hline Mediano & Grande & $-0,039$ \\
\hline
\end{tabular}

* En caso de incumplimiento del supuesto de homogeneidad de medias necesario para la aplicación de la prueba HSD de Tukey (prueba de Levene significativa con una $\mathrm{p} \leq 0.05$ ), se aplica la prueba de comparación de medias con la corrección de Games-Howell $(\mathrm{GH})$. Se han eliminado las comparaciones redundantes.

Diferencias significativas con una $\mathrm{p} \leq 0.001$

Diferencias significativas con una $\mathrm{p} \leq 0.010$

Diferencias significativas con una $\mathrm{p} \leq 0.050$

Fuente: elaboración propia $(N=2163)$

La ubicación de los centros, confirmando en algunos aspectos la tendencia que acabamos de apuntar, también parece que condiciona las formas docentes, principalmente cuando ponemos en contraste los centros urbanos y, de manera más concreta, los situados en Barcelona y su área metropolitana, con las escuelas del ámbito rural. En éstas, parece que los profesores pueden encontrar condiciones que les facilitan mayores opciones de atención a la diversidad, más posibilidades de implicar a los alumnos en las decisiones que afectan al propio proceso educativo, así como de promover la cooperación entre los alumnos y el trabajo en equipo. En este sentido, parece que, en algunos aspectos, la práctica que se desarrolla en el ámbito rural ofrecería unas condiciones más favorables para responder a los retos que nos plantea la Sociedad Red. En algunos aspectos, esta valoración debe tomarse con una cierta prudencia: en algunas de las cuestiones que hemos considerado críticas como la capacidad de incorporar la participación de los padres y otros profesionales en la actividad educativa, la ubicación de los centros no permite identificar diferencias significativas.

Tabla 7a. Comparación de medias en función de la ubicación del centro

\section{(G1 = Barcelona y área metropolitana; G2 = urbanos fuera del área metropo-litana; G3 = semiurb. fuera del área metropolitana; G4 = ámbito rural)}

\begin{tabular}{|l|c|c|c|c|c|}
\hline & $\begin{array}{c}\text { Media G1 } \\
\text { (std. dev.) }\end{array}$ & $\begin{array}{c}\text { Media G2 } \\
\text { (std. dev.) }\end{array}$ & $\begin{array}{c}\text { Media G3 (std. } \\
\text { dev.) }\end{array}$ & $\begin{array}{c}\text { Media G4 (std. } \\
\text { dev.) }\end{array}$ & $\begin{array}{c}\text { Diferencia de } \\
\text { medias* }^{*}\end{array}$ \\
\hline $\begin{array}{l}\text { Promoción de la elaboración del } \\
\text { conocimiento por parte del alumno }\end{array}$ & $4,87(2,12)$ & $4,65(2,17)$ & $4,80(2,12)$ & $4,88(2,06)$ & $\mathrm{F}=0,617$ \\
\hline $\begin{array}{l}\text { Promoción de la participación de los } \\
\text { alumnos en el proceso de aprendizaje }\end{array}$ & $3,59(2,24)$ & $3,36(2,13)$ & $3,78(2,28)$ & $4,09(2,32)$ & $\mathrm{F}=4,748^{\mathrm{b}}$ \\
\hline $\begin{array}{l}\text { Promoción de la participación de padres y } \\
\text { otros profesionales en la actividad docente }\end{array}$ & $2,72(2,24)$ & $2,76(2,00)$ & $2,80(2,24)$ & $3,02(2,33)$ & $\mathrm{F}=1,313$ \\
\hline $\begin{array}{l}\text { Promoción de una organización flexible y } \\
\text { personalizada de la docencia }\end{array}$ & $5,48(2,31)$ & $5,29(2,30)$ & $5,70(2,39)$ & $6,16(2,24)$ & $\mathrm{F}=7,153^{\mathrm{a}}$ \\
\hline $\begin{array}{l}\text { Promoción del trabajo en equipo en la } \\
\text { actividad docente }\end{array}$ & $4,52(2,11)$ & $4,60(2,25)$ & $4,49(2,17)$ & $5,10(2,17)$ & $\mathrm{F}=5,234^{\mathrm{a}}$ \\
\hline $\begin{array}{l}\text { Promoción de la evaluación continua del } \\
\text { proceso de enseñanza y aprendizaje }\end{array}$ & $7,02(2,37)$ & $6,75(2,33)$ & $6,86(2,36)$ & $7,39(2,26)$ & $\mathrm{F}=3,335^{\mathrm{c}}$ \\
\hline $\begin{array}{l}\text { Promoción del uso de materiales diversos } \\
\text { (libros, revistas, materiales multimedia, } \\
\text { páginas web, etc.) }\end{array}$ & $6,80(2,35)$ & $7,02(2,17)$ & $6,89(2,19)$ & $7,04(2,28)$ & $\mathrm{F}=1,130$ \\
\hline
\end{tabular}

* En caso de incumplimiento del supuesto de homogeneidad de medias necesario para la aplicación de la prueba $\mathrm{F}$ (prueba de Levene significativa con una $\mathrm{p} \leq 0.05$ ), se aplica la prueba de comparación de medias con la corrección de Welch (W).

${ }^{a}$ Diferencias significativas con una $\mathrm{p} \leq 0.001$

Diferencias significativas con una $\mathrm{p} \leq 0.010$ 
Fuente: elaboración propia $(N=2163)$

Tabla 7b. Pruebas post-hoc para la comparación de medias dos a dos en función de la ubicación del centro*

\begin{tabular}{|c|c|c|c|}
\hline & Ubieación(H) & Ubieaeión $(J)$ & Dif.de medias (I-J) \\
\hline \multirow{6}{*}{$\begin{array}{l}\text { Promoción de la elaboración del conocimiento por } \\
\text { parte del alumno (Prueba HSD de Tukey) }\end{array}$} & \multirow{3}{*}{$\begin{array}{l}\text { BCN y area } \\
\text { metropolitana }\end{array}$} & $\begin{array}{c}\text { Urbanos fuera del área } \\
\text { metropolitana }\end{array}$ & 0,221 \\
\hline & & $\begin{array}{l}\text { Semiurb. fuera del área } \\
\text { metropolitana }\end{array}$ & 0,066 \\
\hline & & Ámbito rural & $-0,007$ \\
\hline & \multirow{2}{*}{$\begin{array}{l}\text { Urbanos fuera del } \\
\text { área metropolitana }\end{array}$} & $\begin{array}{l}\text { Semiurb. fuera del área } \\
\text { metropolitana }\end{array}$ & $-0,155$ \\
\hline & & Ámbito rural & $-0,228$ \\
\hline & $\begin{array}{l}\text { Semiurb. fuera del } \\
\text { área metropolitana }\end{array}$ & Ámbito rural & $-0,073$ \\
\hline \multirow{6}{*}{$\begin{array}{l}\text { Promoción de la participación de los alumnos en el } \\
\text { proceso de aprendizaje (Prueba HSD de Tukey) }\end{array}$} & \multirow{3}{*}{$\begin{array}{l}\text { BCN y area } \\
\text { metropolitana }\end{array}$} & $\begin{array}{c}\text { Urbanos fuera del área } \\
\text { metropolitana }\end{array}$ & 0,233 \\
\hline & & $\begin{array}{l}\text { Semiurb. fuera del área } \\
\text { metropolitana }\end{array}$ & $-0,191$ \\
\hline & & Ámbito rural & $-0,497^{b}$ \\
\hline & \multirow{2}{*}{$\begin{array}{l}\text { Urbanos fuera del } \\
\text { área metropolitana }\end{array}$} & $\begin{array}{c}\text { Semiurb. fuera del área } \\
\text { metropolitana }\end{array}$ & $-0,424$ \\
\hline & & Ámbito rural & $-0,730^{b}$ \\
\hline & $\begin{array}{l}\text { Semiurb. fuera del } \\
\text { área metropolitana }\end{array}$ & Ámbito rural & $-0,307$ \\
\hline \multirow{6}{*}{$\begin{array}{l}\text { Promoción de la participación de padres y otros } \\
\text { profesionales en la actividad docente (Prueba HSD } \\
\text { de Tukey) }\end{array}$} & \multirow{3}{*}{$\begin{array}{l}\mathrm{BCN} \text { y area } \\
\text { metropolitana }\end{array}$} & $\begin{array}{l}\text { Urbanos fuera del área } \\
\text { metropolitana }\end{array}$ & $-0,040$ \\
\hline & & $\begin{array}{l}\text { Semiurb. fuera del área } \\
\text { metropolitana }\end{array}$ & $-0,087$ \\
\hline & & Ámbito rural & $-0,305$ \\
\hline & \multirow{2}{*}{$\begin{array}{l}\text { Urbanos fuera del } \\
\text { área metropolitana }\end{array}$} & $\begin{array}{l}\text { Semiurb. fuera del área } \\
\text { metropolitana }\end{array}$ & $-0,047$ \\
\hline & & Ámbito rural & $-0,265$ \\
\hline & $\begin{array}{l}\text { Semiurb. fuera del } \\
\text { área metropolitana }\end{array}$ & Ámbito rural & $-0,217$ \\
\hline \multirow{6}{*}{$\begin{array}{l}\text { Promoción de una organización flexible y } \\
\text { personalizada de la docencia (Prueba HSD de Tukey) }\end{array}$} & \multirow{3}{*}{$\begin{array}{l}\text { BCN y area } \\
\text { metropolitana }\end{array}$} & $\begin{array}{c}\text { Urbanos fuera del área } \\
\text { metropolitana }\end{array}$ & 0,189 \\
\hline & & $\begin{array}{c}\text { Semiurb. fuera del área } \\
\text { metropolitana }\end{array}$ & $-0,222$ \\
\hline & & Ámbito rural & $-0,683^{a}$ \\
\hline & \multirow{2}{*}{$\begin{array}{l}\text { Urbanos fuera del } \\
\text { área metropolitana }\end{array}$} & $\begin{array}{l}\text { Semiurb. fuera del área } \\
\text { metropolitana }\end{array}$ & $-0,411$ \\
\hline & & Ámbito rural & $-0,872^{\mathrm{a}}$ \\
\hline & $\begin{array}{l}\text { Semiurb. fuera del } \\
\text { área metropolitana }\end{array}$ & Ámbito rural & $-0,461$ \\
\hline \multirow{6}{*}{$\begin{array}{l}\text { Promoción del trabajo en equipo en la actividad } \\
\text { docente (Prueba HSD de Tukey) }\end{array}$} & \multirow{3}{*}{$\begin{array}{l}\text { BCN y area } \\
\text { metropolitana }\end{array}$} & $\begin{array}{l}\text { Urbanos fuera del área } \\
\text { metropolitana }\end{array}$ & $-0,080$ \\
\hline & & $\begin{array}{l}\text { Semiurb. fuera del área } \\
\text { metropolitana }\end{array}$ & 0,028 \\
\hline & & Ámbito rural &,$- 574^{\mathrm{a}}$ \\
\hline & \multirow[t]{2}{*}{$\begin{array}{l}\text { Urbanos fuera del } \\
\text { área metropolitana }\end{array}$} & $\begin{array}{l}\text { Semiurb. fuera del área } \\
\text { metropolitana }\end{array}$ & 0,108 \\
\hline & & Ámbito rural & $-0,494$ \\
\hline & $\begin{array}{l}\text { Semiurb. fuera del } \\
\text { área metropolitana }\end{array}$ & Ámbito rural &,$- 602^{b}$ \\
\hline
\end{tabular}


Promoción de la evaluación continua del proceso de enseñanza y aprendizaje (Prueba HSD de Tukey)

\begin{tabular}{|c|c|c|}
\hline \multirow{3}{*}{$\begin{array}{l}\mathrm{BCN} \text { y area } \\
\text { metropolitana }\end{array}$} & $\begin{array}{l}\text { urvanus ı uera ues area } \\
\text { metropolitana }\end{array}$ & 0,267 \\
\hline & $\begin{array}{c}\text { Semiurb. fuera del área } \\
\text { metropolitana }\end{array}$ & 0,157 \\
\hline & Ámbito rural & $-0,376$ \\
\hline \multirow{2}{*}{$\begin{array}{l}\text { Urbanos fuera del } \\
\text { área metropolitana }\end{array}$} & $\begin{array}{c}\text { Semiurb. fuera del área } \\
\text { metropolitana }\end{array}$ & $-0,110$ \\
\hline & Ámbito rural & $-0,643^{\mathrm{c}}$ \\
\hline $\begin{array}{l}\text { Semiurb. fuera del } \\
\text { área metropolitana }\end{array}$ & Ámbito rural & $-0,532^{c}$ \\
\hline \multirow{3}{*}{$\begin{array}{l}\text { BCN y area } \\
\text { metropolitana }\end{array}$} & $\begin{array}{c}\text { Urbanos fuera del área } \\
\text { metropolitana }\end{array}$ & $-0,226$ \\
\hline & $\begin{array}{c}\text { Semiurb. fuera del área } \\
\text { metropolitana }\end{array}$ & $-0,096$ \\
\hline & Ámbito rural & $-0,240$ \\
\hline \multirow{2}{*}{$\begin{array}{l}\text { Urbanos fuera del } \\
\text { área metropolitana }\end{array}$} & $\begin{array}{c}\text { Semiurb. fuera del área } \\
\text { metropolitana }\end{array}$ & 0,130 \\
\hline & Ámbito rural & $-0,014$ \\
\hline $\begin{array}{l}\text { Semiurb. fuera del } \\
\text { área metropolitana }\end{array}$ & Ámbito rural & $-0,144$ \\
\hline
\end{tabular}

* En caso de incumplimiento del supuesto de homogeneidad de medias necesario para la aplicación de la prueba HSD de Tukey (prueba de Levene significativa con una $\mathrm{p} \leq 0.05$ ), se aplica la prueba de comparación de medias con la corrección de Games-Howell (GH). Se han eliminado las comparaciones redundantes.

Diferencias significativas con una $\mathrm{p} \leq 0.001$

Diferencias significativas con una $\mathrm{p} \leq 0.010$

Diferencias significativas con una $\mathrm{p} \leq 0.050$

Fuente: elaboración propia $(N=2163)$

4.5.4. Diferencias en función de la formación inicial del profesorado.

En último término, aparte de estas diferencias entre centros y etapas, también nos fijaremos en algunos de los factores que permiten distinguir la actividad docente desde un punto de vista individual.

En primer lugar, puede ser interesante intentar una aproximación a la incidencia que puede tener la formación inicial del profesorado (ver tablas $8 \mathrm{a}$ y 8b) en la manera cómo éste interpreta su práctica pedagógica. Las diferencias que se pueden apreciar a partir de la valoración que nos ofrecen los profesores, se decantan del lado de los maestros en una buena parte de los aspectos que hemos considerado más interesantes para dar respuesta a los requerimientos de una sociedad informacional. De hecho, los maestros son los que más a menudo se identifican con los planteamientos que más favorecen la participación activa del estudiante y la posibilidad de tomar decisiones sobre su proceso de aprendizaje: las diferencias en la comparación de éstos con los licenciados se producen significativamente a favor de los maestros en todos los casos.

Tabla 8a. Comparación de medias en función de la formación inicial

(G1 = téc. sup. y diplomados; G2 = licenciados; G3 = tercer ciclo)

\begin{tabular}{|l|c|c||c|c|}
\hline & $\begin{array}{c}\text { Media G1 } \\
\text { (std. dev.) }\end{array}$ & $\begin{array}{c}\text { Media G2 (std. } \\
\text { dev.) }\end{array}$ & $\begin{array}{c}\text { Media G3 (std. } \\
\text { dev.) }\end{array}$ & $\begin{array}{c}\text { Diferencia de } \\
\text { medias* }\end{array}$ \\
\hline $\begin{array}{l}\text { Promoción de la elaboración del conocimiento por parte } \\
\text { del alumno }\end{array}$ & $5,08(2,21)$ & $4,66(2,00)$ & $5,13(2,25)$ & $\mathrm{W}=10,799^{\mathrm{a}}$ \\
\hline $\begin{array}{l}\text { Promoción de la participación de los alumnos en el } \\
\text { proceso de aprendizaje }\end{array}$ & $3,83(2,27)$ & $3,53(2,20)$ & $3,75(2,37)$ & $\mathrm{F}=4,217^{\mathrm{c}}$ \\
\hline
\end{tabular}




\begin{tabular}{|c|c|c|c|c|}
\hline $\begin{array}{l}\text { riomocion de ia participacion ue paures y uirus } \\
\text { profesionales en la actividad docente }\end{array}$ & $3,37(2,29)$ & $2,45(2,13)$ & $2,51(2,15)$ & $\mathrm{W}=38,932^{\mathrm{a}}$ \\
\hline $\begin{array}{l}\text { Promoción de una organización flexible y personalizada de } \\
\text { la docencia }\end{array}$ & $6,04(2,15)$ & $5,32(2,37)$ & $5,57(2,34)$ & $\mathrm{W}=23,144^{\mathrm{a}}$ \\
\hline Promoción del trabajo en equipo en la actividad docente & $5,09(2,10)$ & $4,28(2,11)$ & $4,50(2,10)$ & $\mathrm{F}=32,462^{\mathrm{a}}$ \\
\hline $\begin{array}{l}\text { Promoción de la evaluación continua del proceso de } \\
\text { enseñanza y aprendizaje }\end{array}$ & $7,40(2,19)$ & $6,75(2,42)$ & $7,18(2,28)$ & $\mathrm{W}=18,644^{\mathrm{a}}$ \\
\hline $\begin{array}{l}\text { Promoción del uso de materiales diversos (libros, revistas, } \\
\text { materiales multimedia, páginas web, etc.) }\end{array}$ & $7,14(2,15)$ & $6,62(2,37)$ & $7,02(2,34)$ & $\mathrm{W}=12,436^{\mathrm{a}}$ \\
\hline
\end{tabular}

* En caso de incumplimiento del supuesto de homogeneidad de medias necesario para la aplicación de la prueba $\mathrm{F}$ (prueba de Levene significativa con una $\mathrm{p} \leq 0.05$ ), se aplica la prueba de comparación de medias con la corrección de Welch (W).

${ }^{a}$ Diferencias significativas con una $\mathrm{p} \leq 0.001$

Diferencias significativas con una $\mathrm{p} \leq 0.010$

Diferencias significativas con una $\mathrm{p} \leq 0.050$

Fuente: elaboración propia $(N=2163)$

Por otro lado, no siempre se producen diferencias significativas cuando comparamos a los profesores que disponen de formación de tercer ciclo con los maestros, pero cuando las encontramos nuevamente son estos últimos los que consideran su práctica más flexible, favorable a la colaboración entre los alumnos y abierta a la participación. Coincidiendo en buena medida con las diferencias en función de la etapa que señalábamos antes, también son los maestros los que se atribuyen con mayor frecuencia las formas de trabajo que promueven con más intensidad la colaboración, el trabajo en equipo y también las prácticas docentes más abiertas a la participación externa. Esta situación, que podemos vincular a la incidencia de la formación inicial de los maestros en su práctica docente, pone de manifiesto en qué medida este componente pedagógico constituye un factor crítico en la formación del profesorado de secundaria.

Tabla 8b. Pruebas post-hoc para la comparación de medias dos a dos en función de la formación inicial*

\begin{tabular}{|c|c|c|c|}
\hline & Formación (I) & Formación (J) & Dif. de medias (I-J) \\
\hline \multirow{3}{*}{$\begin{array}{l}\text { Promoción de la elaboración del conocimiento por parte } \\
\text { del alumno (Prueba de Games Howell) }\end{array}$} & \multirow{2}{*}{$\begin{array}{l}\text { Tec. sup. y } \\
\text { diplomados }\end{array}$} & Licenciados & $0,423^{\mathrm{a}}$ \\
\hline & & Tercer ciclo & $-0,051$ \\
\hline & Licenciados & Tercer ciclo & $-0,474^{b}$ \\
\hline \multirow{3}{*}{$\begin{array}{l}\text { Promoción de la participación de los alumnos en el } \\
\text { proceso de aprendizaje (Prueba HSD de Tukey) }\end{array}$} & \multirow{2}{*}{$\begin{array}{l}\text { Tec. sup. y } \\
\text { diplomados }\end{array}$} & Licenciados & $0,303^{b}$ \\
\hline & & Tercer ciclo & 0,080 \\
\hline & Licenciados & Tercer ciclo & $-0,223$ \\
\hline \multirow{3}{*}{$\begin{array}{l}\text { Promoción de la participación de padres y otros } \\
\text { profesionales en la actividad docente (Prueba de Games } \\
\text { Howell) }\end{array}$} & \multirow{2}{*}{$\begin{array}{l}\text { Tec. sup. y } \\
\text { diplomados }\end{array}$} & Licenciados & $0,924^{\mathrm{a}}$ \\
\hline & & Tercer ciclo & $0,860^{\mathrm{a}}$ \\
\hline & Licenciados & Tercer ciclo & $-0,065$ \\
\hline \multirow{3}{*}{$\begin{array}{l}\text { Promoción de una organización flexible y personalizada de } \\
\text { la docencia (Prueba de Games Howell) }\end{array}$} & \multirow{2}{*}{$\begin{array}{l}\text { Tec. sup. y } \\
\text { diplomados }\end{array}$} & Licenciados & $0,727^{\mathrm{a}}$ \\
\hline & & Tercer ciclo & $0,476^{\mathrm{c}}$ \\
\hline & Licenciados & Tercer ciclo & $-0,251$ \\
\hline \multirow{3}{*}{$\begin{array}{l}\text { Promoción del trabajo en equipo en la actividad docente } \\
\text { (Prueba HSD de Tukey) }\end{array}$} & \multirow{2}{*}{$\begin{array}{l}\text { Tec. sup. y } \\
\text { diplomados }\end{array}$} & Licenciados & $0,807^{\mathrm{a}}$ \\
\hline & & Tercer ciclo & $0,589^{\mathrm{a}}$ \\
\hline & Licenciados & Tercer ciclo & $-0,219$ \\
\hline \multirow{3}{*}{$\begin{array}{l}\text { Promoción de la evaluación continua del proceso de } \\
\text { enseñanza y aprendizaje (Prueba de Games Howell) }\end{array}$} & \multirow{2}{*}{$\begin{array}{l}\text { Tec. sup. y } \\
\text { diplomados }\end{array}$} & Licenciados & $0,657^{\mathrm{a}}$ \\
\hline & & Tercer ciclo & 0,220 \\
\hline & Licenciados & Tercer ciclo & $-0,437^{\mathrm{c}}$ \\
\hline Promoción del uso de materiales diversos (libros, revistas, & Tec. sup. y & Licenciados & $0,520^{\mathrm{a}}$ \\
\hline
\end{tabular}


materiales multimedia, páginas web, etc.) (Prueba de Games Howell)

\begin{tabular}{|c|} 
diplomados \\
\hline Licenciados
\end{tabular}

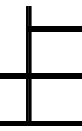

Tercer ciclo

0,126

Tercer ciclo

$-0,395$

* En caso de incumplimiento del supuesto de homogeneidad de medias necesario para la aplicación de la prueba HSD de

Tukey (prueba de Levene significativa con una $\mathrm{p} \leq 0.05$ ), se aplica la prueba de comparación de medias con la corrección de Games-Howell (GH). Se han eliminado las comparaciones redundantes.

Diferencias significativas con una $\mathrm{p} \leq 0.001$

Diferencias significativas con una $\mathrm{p} \leq 0.010$

Diferencias significativas con una $\mathrm{p} \leq 0.050$

Fuente: elaboración propia $(N=2163)$

\subsubsection{Diferencias en función de la edad del profesorado.}

En último término, para cerrar esta aproximación general a algunos de los principales aspectos que distinguen el conjunto del profesorado, puede ser relevante poner la atención en la medida en que las prácticas que estamos considerando más valiosas se están incorporando a los centros de la mano de los profesores más jóvenes (ver tabla 9).

La tendencia que hemos podido identificar, en este sentido, nos permite ser optimistas: aunque la edad no siempre permite establecer diferencias importantes entre el profesorado, los profesores más jóvenes (menores de treinta y cinco años) son los que en la mayoría de los casos se identifican con las formas de docencia más centradas en el estudiante, los que se atribuyen los estilos docentes más flexibles y, por lo tanto, los más propicios a la personalización. Únicamente cuando nos referimos al grado de participación en la docencia son los profesores más veteranos los que se atribuyen una posición de mayor apertura. Aunque las diferencias no siempre son relevantes, podríamos interpretar que los estilos docentes a que concedemos mayor potencialidad parece que se incorporan a los centros a través de la práctica de los profesores más jóvenes.

\section{Tabla 9. Comparación de medias en función de la edad}

$$
\text { (G1 = hasta } 35 \text { años; G2 = } 36 \text { años o más) }
$$

\begin{tabular}{|c|c|c|c|}
\hline & \begin{tabular}{|c|}
$\begin{array}{c}\text { Media G1 (std. } \\
\text { dev.) }\end{array}$ \\
\end{tabular} & $\begin{array}{c}\text { Media G2 (std. } \\
\text { dev.) }\end{array}$ & $\begin{array}{c}\begin{array}{c}\text { Diferencia de } \\
\text { medias* }\end{array} \\
\end{array}$ \\
\hline Promoción de la elaboración del conocimiento por parte del alumno & $5,03(2,10)$ & $4,76(2,13)$ & $\mathrm{F}=7,305^{\mathrm{b}}$ \\
\hline Promoción de la participación de los alumnos en el proceso de aprendizaje & $3,93(2,32)$ & $3,53(2,12)$ & $\mathrm{F}=14,436^{\mathrm{a}}$ \\
\hline $\begin{array}{l}\text { Promoción de la participación de padres y otros profesionales en la } \\
\text { actividad docente }\end{array}$ & $2,59(2,20)$ & $2,85(2,23)$ & $\mathrm{F}=5,813^{\mathrm{c}}$ \\
\hline Promoción de una organización flexible y personalizada de la docencia & $5,93(2,39)$ & $5,43(2,28)$ & $\mathrm{F}=20,465^{\mathrm{a}}$ \\
\hline Promoción del trabajo en equipo en la actividad docente & $4,54(2,29)$ & $4,60(2,08)$ & $\mathrm{W}=0,304$ \\
\hline $\begin{array}{l}\text { Promoción de la evaluación continua del proceso de enseñanza y } \\
\text { aprendizaje }\end{array}$ & $7,29(2,22)$ & $6,90(2,39)$ & $\mathrm{F}=13,078^{\mathrm{a}}$ \\
\hline $\begin{array}{l}\text { Promoción del uso de materiales diversos (libros, revistas, materiales } \\
\text { multimedia, páginas web, etc.) }\end{array}$ & $6,93(2,39)$ & $6,81(2,26)$ & $\mathrm{F}=1,079$ \\
\hline \multicolumn{4}{|c|}{$\begin{array}{l}\text { * En caso de incumplimiento del supuesto de homogeneidad de medias necesario para la aplicación de la prueba F (prueba } \\
\text { de Levene significativa con una } \mathrm{p} \leq 0.05 \text { ), se aplica la prueba de comparación de medias con la corrección de Welch (W). }\end{array}$} \\
\hline \multicolumn{4}{|l|}{${ }^{a}$ Diferencias significativas con una $\mathrm{p} \leq 0.001$} \\
\hline \multicolumn{4}{|l|}{${ }^{b}$ Diferencias significativas con una $\mathrm{p} \leq 0.010$} \\
\hline Diferencias significativas con una $\mathrm{p} \leq 0.050$ & & & \\
\hline
\end{tabular}


La formación inicial de las nuevas generaciones de maestros continúa apareciendo, en este sentido, como una cuestión clave para acercar las aulas a las demandas de la Sociedad Red, pero, seguramente, todavía es más crítica la formación continuada: aunque la experiencia profesional permite apreciar diferencias en pocos aspectos, cuando las encontramos, las prácticas más personalizadas, las que ofrecen mayores oportunidades de participación a los alumnos no se sitúan más cerca de los profesores que acumulan más años de antigüedad en el centro. Que la experiencia, en este caso, no sea un grado, pone sobre la mesa un reto fundamental que deberemos encarar decididamente si queremos un sistema educativo que pueda ser capaz de responder con eficiencia a la complejidad de demandas que le plantea a la sociedad actual.

\section{Conclusiones}

Vivimos en una Sociedad Red, con una creciente economía del conocimiento en que las mayores oportunidades están al alcance de las personas que saben trabajar de forma flexible y creativa, de las que han aprendido a colaborar en un marco de inestabilidad social creciente (Carnoy, 2002). Los profesores tienen que comprender y saber moverse en este marco si pretenden poder preparar a sus alumnos para este mundo que, sobre la base del informacionalismo (Castells, 2001a), está experimentando profundas transformaciones (Castells et al., 2003, para un análisis del caso de Cataluña).

Desgraciadamente, una buena parte de los debates en torno a los cambios necesarios para acercar la actividad de la escuela al contexto social actual, han quedado mayoritariamente centrados en torno a una cuestión que, a nuestro entender, tendría que ser considerada secundaria: la incorporación de las TIC y, especialmente, de la red al ámbito educativo. Esta introducción es, obviamente, un tema fundamental del que parte nuestra propia investigación (Mominó et al. 2004). Pero éstas, como cualquier otra tecnología, al fin y al cabo son herramientas e instrumentos, que no pueden desvincular sus bondades de las finalidades para las que se utilizan ni de los propios contextos donde se introducen (Fischer, 1992, para un interesante acercamiento en el campo del estudio de la tecnología y el cambio social). En este sentido, y recuperando esta especial sensibilidad por el contexto, la clave para la verdadera incorporación de la red a los procesos de enseñanza y aprendizaje no se explicaría sólo por las propias características de la tecnología. Es decir, a pesar de su alta potencialidad, la cuestión fundamental se refiere tanto a las facilidades que ofrece la propia tecnología para el acceso y la recombinación de la información, o para el desarrollo de nuevas oportunidades de comunicación, de interacción y de representación del conocimiento. Contrariamente, el verdadero potencial de la red es modulado directamente por la singularidad de la práctica pedagógica en que se incorpora. Depende del contexto en que se inserta (Pierson, 2001) más que de su supuesta capacidad intrínseca de transformación (Becker, 2000).

La cuestión es que, tal como muestra esta investigación, parece que el papel preponderante del docente a menudo toma el protagonismo imprescindible que tendría que tener el alumno en el mismo proceso de aprender. El profesorado reconoce que ofrece pocas oportunidades a los alumnos de participar en las decisiones que afectan a su implicación en este proceso y los responsables pedagógicos de las diferentes etapas educativas confirman este punto de vista. Por otra parte, parece que los profesores todavía continúan afrontando de manera bastante aislada los retos cotidianos que les plantea su actividad en el aula en bastantes casos. En la práctica, hay poco espacio para la participación de los padres y madres y, en general, de la comunidad educativa. Así mismo la actividad del aula parece acercarse poco a un enfoque basado en la cooperación y el trabajo en equipo (Meneses y Mominó, forthcoming, para una visión de conjunto de estos aspectos). Además, a pesar del valor fundamental que concedemos a la individualización por su valor estratégico ante el reto ineludible de la atención a la diversidad, el profesorado todavía tendría que tomar de manera más decidida el camino hacia formas más personalizadas de la acción docente. En cambio, la función formativa o reguladora de la evaluación parece haberse incorporado en mayor medida a la práctica docente cotidiana como instrumento de mejora del propio proceso de enseñanza y aprendizaje. 
Enseñar en esta sociedad supone, más que nunca, promover el aprendizaje significativo, "la creatividad y la inventiva de los estudiantes; apostar por la investigación, el trabajo en red y en equipo; y la formación profesional permanente de los docentes; y promover la resolución de problemas, la asunción de riesgos, la confianza en el proceso de colaboración, la habilidad para enfrentarse al cambio y el compromiso con la mejora continua como organizaciones" (Hargreaves, 2003, 12). Desde este punto de vista, la incorporación de las TIC y la red al ámbito educativo proporciona nuevas oportunidades para la adaptación de las prácticas educativas que, en este contexto, se encuentran en la necesidad de redefinir su dinámica i sus finalidades.

En una Sociedad Red como la nuestra, la mejora de calidad de la enseñanza en las aulas no se tendría que esperar por efecto del impacto de la introducción de internet a las aulas. La incidencia de las tecnologías en este ámbito, de las posibilidades de acceso y procesamiento de la información, comunicación e interactividad, depende directamente de las características del modelo pedagógico donde se introduce su uso y, en consecuencia, de la manera cómo se estructuran los entornos de formación con la incorporación de la red. Es desde este supuesto que nos hemos aproximado a algunas de las características de la acción docente, desde la perspectiva del profesorado, para entender las posibilidades que se abren con la incorporación de internet a la práctica pedagógica. Internet, como cualquier otra tecnología, se puede poner al servicio de las prácticas docentes más contrapuestas. Solamente algunas que todavía no parecen suficientemente presentes en las aulas, promueven el mayor protagonismo del estudiante cuando de lo que se trata es de dar el salto, que la tecnología no permite despreciar, entre la información y el conocimiento.

\section{Bibliografia}

BECKER, H. J. (2000): Findings from the teaching, learning, and computing survey: Is Larry Cuban right? TLC 12 Special Report. Center for Research on Information Technology and Organizations. Consultado el 1 de marzo, 2006, desde CRITO, http://www.crito.uci.edu/tlc/findings/ccsso.pdf

BENTLEY, T. (1998): Life Beyond the Classroom. London, Rouledge.

CARNOY, M. (2002): Sustaining the new economy. Work, family, and community in the information age. New York, Russell Sage \& Harvard University Press.

CARNOY, M. (2004): Las TIC en la enseñanza: posibilidades y retos. Consultado el 1 de marzo, 2006, desde la UOC, http://www.uoc.edu/inaugural04/dt/esp/carnoy1004.pdf

CASTELLS, M. (2000): The Rise of the Network Society (2nd ed). Malden (MA), Blackwell Publishers.

CASTELLS, M. (2001a): Informacionalismo y la Sociedad Red, en HIMANEN, P. (Ed.) La ética del hacker y el espíritu de la era de la información. Barcelona, Destino, 169-191.

CASTELLS, M. (2001b): Internet y la Sociedad Red. Lección inaugural del programa de doctorado de sobre sociedad de la información y el conocimiento. Consultado el 1 de marzo, 2006, desde la UOC, http://www.uoc.edu/web/esp/articles/castells/castellsmain.html

CASTELLS, M. (2001c): La galaxia internet. Reflexiones sobre internet, empresa y sociedad. Barcelona, Plaza \& Janes.

CASTELLS, M., TUBELLA, I., SANCHO, T., DÍAZ, I. y WELLMAN, B. (2003): La societat xarxa a Catalunya. Barcelona, Rosa dels Vents.

CETRON, M. y GAYLE, M. (1991): Educational Renaissance, our schools at the turn of the 21st Century. New York, St. Martin's Press. 
COHEN, D. (1987): Educational technology, policy, and practice. Educational Evaluation and Policy Analysis, 9, 153-170.

COLL, C. y ONRUBIA,J. (1996) La construcción de significados compartidos en el aula: actividad conjunta y dispositivos semióticos en el control y seguimiento mutuo entre profesor y alumnos. En COLL, C. y EDWARDS (Eds.) Enseñanza, aprendizaje y discurso en el aula. Aproximaciones al estudio del discurso educacional. Madrid, Infancia y Aprendizaje, 53-73.

COLLER, X. (1997): La empresa flexible. Estudio sociológico del impacto de la flexibilidad en el proceso de trabajo. Madrid, Siglo XXI / Centro de Investigaciones Sociológicas.

CUBAN, L. (1986): Teachers and machines. The classroom use of technology since 1920. New York, Teachers College Press.

CUBAN, L. (2003): Oversold and underused: Computers in the classroom. Cambridge, Harvard University Press.

CUBAN, L., KIRKPATRICK, H. y PECK, C. (2001): High access and low use of technologies in high school classrooms: explaining an apparent paradox. American Educational Research Journal, 38(4), 813-834.

DELORS, J. (1996). La educación encierra un tesoro. Barcelona, UNESCO.

DEMETRIADIS, S., BARBAS, A., MOLOHIDES, A., PALAIGEORGIOU, G., PSILLOS, D., VLAHAVAS, I., TSOUKALAS, I. y POMBORTSIS, A. (2003): "Cultures in negotiation": teachers' acceptance / resistance attitudes considering the infusion of technology into schools. Computers \& Education archive, 41(1), 19-37.

FISCHER, C. S. (1992): America calling: a social history of the telephone to 1940. Berkeley, University of California Press.

GLASS, G. V., PECKHAM, P. D. y SANDERS, J. R. (1972): Consequences of failure to meet assumptions underlying the fixed effects analysis of variance and covariance. Review of Educational Research, 42, 237-288.

HANNAFIN, R. D. y SAVENYE, W. C. (1993): Technology in the classroom: the teacher's new role and resistance to it. Educational Technology, 33(6), 26-31.

HARGREAVES, A. (2003): Enseñar en la sociedad del conocimiento. Barcelona, Octaedro.

HARRISON, C., COMBER, C., FISCHER, T., HAW, K., LEWIN, C., LUNZER, E., McFARLANE, A., MAVERS, D., SCRIMSHAW, P., SOMEKH, B., y WATLING, R. (2002): ImpaCT2. The impact of information and communication technologies on pupil learning and attainment. ICT in Schools

Research and Evaluation Series, 7. Coventry, British Educational Communications and Technology

Agency. Consultado el 1 de marzo, 2006, desde BECTA,

http://www.becta.org.uk/page_documents/research/ImpaCT2_strand1_report.pdf

JACCARD, J. y WAN, C. K. (1996): LISREL approaches to interaction effects in multiple regression. Thousand Oaks (CA), Sage Publications.

JONASSEN, D. H. (1999): Computers as mindtools for schools: engaging critical thinking (2nd ed.). Englewood Cliffs (NJ), Prentice Hall.

LÉVY, P. (1998): Cibercultura. El segon diluvi? Barcelona, Proa.

MARCHESI, A. (2000): ¿Formación humanística o científica? En MARCHESI, A. (Ed.) 
Controversias en la educación española. Madrid, Alianza Editorial, 163-202.

MARCHESI, A. y MARTÍN, E. (1998): Calidad de la enseñanza en tiempos de cambio. Madrid, Alianza.

MARTÍNEZ, M., BUJONS, C., FLECK, M. y PRATS, E. (2001): Un lugar llamado escuela. En la sociedad de la información y de la diversidad. Barcelona, Ariel.

MENESES, J. y MOMINÓ, J. M. (forthcoming): La generación de capital social en el sistema educativo no universitario: un análisis del desarrollo comunitario de las escuelas de Cataluña.

MILLER, R. G. (1981): Simultaneous statistical inference (2nd. ed.). New York, McGraw-Hill.

MOMINÓ, J. M., SIGALÉS, C., FORNIELES, A., GUASCH, T. y ESPASA, A. (2004): L'escola a la Societat Xarxa: internet en l'àmbit educatiu no universitari. Informe de recerca. Consultado el 1 de marzo, 2006, desde IN3, http://www.uoc.edu/in3/pic/cat/pic31.html

MONEREO, C. (2005): Internet i competencias bàsicas. Barcelona, Graó.

MORIN, E. (2001): La tête bien faite: repenser la réforme, réformer la pensée. Paris, Senil.

NIEDERHAUSER, D. S. y STODDART, T. (2001): Teachers' instructional perspectives and use of educational software. Teaching and Teacher Education, 17(1), 15-31.

OCDE (2000): Knowdlege management in the learning society. Education and Skills. París, OCDE.

OCDE (2001): Learning to change: ICT in Schools. París, OCDE

PAPERT, S. (1993): The children's machine: rethinking school in the age of the computer. New York, Basic Books, Inc.

PÉREZ, J. M. (2000): Las escuelas y la enseñanza en la sociedad de la información, en PÉREZ, J. M. (Ed.) Comunicación y educación en la sociedad de la información. Barcelona, Paidós Ibérica, 37-57.

PIERSON, M. E. (2001): Technology integration practice as a function of pedagogical expertise. Journal of Research on Computing in Education, 33(4), 413-430.

PISAPIA, J. (1994): Teaching with technology: roles and styles. Research Brief \#5. Metropolitan Educational Research Consortium, Virginia Commonwealth University. Consultado el 1 de marzo, 2006, desde MERC, http://www.soe.vcu.edu/merc/briefs/brief5.htm

SMEETS, E. (2004): Does ICT contribute to powerful learning environments in primary education? Computers and Education, 44, 343-355.

SMEETS, E. y MOOIJ, T. (2001): Pupil-centred learning, ICT, and teacher behaviour: observations in educational practice. British Journal of Educational Technology, 32(4), 403-418.

SUSMAN, E. B. (1998): Co-operative learning: a review of factors that increase the effectiveness of computer-based instruction. Journal of Educational Computing Research, 18(4), 303-322.

TOMARKEN, A. J. y SERLIN, R. C. (1986): Comparison of ANOVA alternatives under variance heterogeneity and specific noncentraly structures. Psychological Bulletin, 99, 90-99.

TOOTHAKER, L. (1991): Multiple comparisons for researchers. Newbury Park (CA), Sage. 
Notas:

[1] Con el apoyo del Departament d'Universitats, Recerca i Societat de la Informació de la Generalitat de Catalunya y del Fondo Social Europeo.

(C) Ediciones Universidad de Salamanca 